\title{
Cytoplasmic p21 induced by p65 prevents doxorubicin-induced cell death in pancreatic carcinoma cell line
}

\author{
YingQi Zhou ${ }^{1 \dagger}$, Gang $\mathrm{Li}^{1 \dagger}$, Yuan $\mathrm{Ji}^{2}$, Chen $\mathrm{Liu}^{3}$, JingPing Zhu ${ }^{3}$ and YanJun $\mathrm{Lu}^{3^{*}}$
}

\begin{abstract}
Background: Studies have shown the existence of p21 induction in a p53-dependent and -independent pathway. Our previous study indicates that DOX-induced p65 is able to bind the p21 promoter to activate its transactivation in the cells.
\end{abstract}

Methods: Over-expression and knock-down experiments were performed in Human Pancreatic Carcinoma (PANC1) cells. Cell cycle and cell death related proteins were assessed by Western Blotting. Cytotoxicity assay was checked by CCK-8 kit. Cell growth was analyzed by flow cytometers.

Results: Here we showed that over-expression of p65 decreased the cytotoxic effect of DOX on PANC1 cells, correlating with increased induction of cytoplasmic p21. We observed that pro-caspase-3 physically associated with cytoplasmic p21, which may be contribution to prevent p21 translocation into the nucleus. Our data also suggested that no clear elevation of nuclear p21 by p65 provides a survival advantage by progression cell cycle after treatment of DOX. Likewise, down-regulation of p65 expression enhanced the cytotoxic effect of DOX, due to a significant decrease of mRNA levels of anti-apoptotic genes, such as the cellular inhibitor of apoptosis-1 (c-IAP1), and the long isoform of B cell leukemia/lymphoma-2 (Bcl-2), leading to efficient induction of caspase-3 cleavage in the cells. More, we present evidence that over-expression of $\mathrm{p} 53$ or p53/p65 in the PANC1 cells were more sensitive to DOX treatment, correlated with activation of caspase-3 and clear elevation of nuclear p21 level. Our previous data suggested that expression of p21 increases Gefitinib-induced cell death by blocking the cell cycle at the G1 and G2 phases. The present findings here reinforced this idea by showing p21's ability of potentiality of DOX-induced cell death correlated with its inhibition of cell cycle progression after over-expression of p53 or p53/p65.

Conclusion: Our data suggested p65 could increase p53-mediated cell death in response to DOX in PANC1 cells. Thus, it is worth noting that in p53 null or defective tumors, targeting in down-regulation of p65 may well be useful, leading to the potentiality of chemotherapeutic drugs.

Keywords: p21, p65, p53, caspase-3, DOX, PANC1

\section{Background}

Pancreatic carcinoma is one of the most leading causes of cancer mortality in the worldwide. Due to the aggressive nature of the disease and the difficulties in diagnosis, the overall 5-year survival rate of pancreatic carcinoma is less than $5 \%[1,2]$. Pancreatic cancer has

\footnotetext{
* Correspondence: yanjunlu@hotmail.com

+ Contributed equally

${ }^{3}$ Laboratory of Cancer Research, Tongji University School of Medicine, 1239

Siping Road, Shanghai 200092, China

Full list of author information is available at the end of the article
}

been reported to be resistant to most of chemotherapeutic drugs. The novel approaches for the treatment of pancreatic cancer are necessary to improve the survival rate.

Nuclear factor $-\kappa \mathrm{B}(\mathrm{NF} \kappa \mathrm{B}) / \mathrm{p} 65$ are a transcriptional factor involved in the response to various stimuli and play a central role in inflammatory reactions. P65 generally exists as an inactive dimer sequestered in the cytoplasm by an inhibitor protein termed $\mathrm{I} \kappa \mathrm{B}$. Activation of p65 translocate into the nucleus, where it binds to the target genes, and promotes transcription [3].

\section{Ciomed Central}


In response to DNA damage induced by cytotoxic agents, the tumor suppressor p53 accumulates and functions as a sequence-specific DNA-binding protein, which positively regulates expression of several genes, including p21 (waf1/cip1/sdl1) [4]. P21 is an important cellular checkpoint protein for G1 and G2 arrest [5-7].

It is widely accepted that p21 is induced by p53dependent and p53-independent pathway. We and other group reported that a novel potential $\mathrm{NF} \kappa \mathrm{B} / \mathrm{p} 65$ binding site is at position -2008 of the p21 promoter and the binding of $\mathrm{NF} \kappa \mathrm{B} / \mathrm{p} 65$ protein to this $\kappa \mathrm{B}$ site results in transactivation of $\mathrm{p} 21$ promoter by $\mathrm{p} 65[8,9]$.

Doxorubicin (DOX) is the most widely used chemotherapy agent in treatment of tumor. DOX targets DNA topoisomerase II enzyme activity, which involves sequential DNA binding, cleavage of DNA phosphodiester backbone and subsequently causes DNA breaks. Recent data suggest that DOX-induced cell death is interconnected with the machinery of cell cycle control. Progression through G1 or G2 phase and entry into the $\mathrm{S}$ or $\mathrm{M}$ phase are tightly regulated by cyclin-dependent kinases (CDKs). Alterations in cell cycle control are a universal feature of cancers. Our previous data suggested that the abnormal progression of cell cycle related with p21 is responsible for DOX-induced cell death [10-13].

Genetic and biochemical studies indicate that DOXinduced cell death is triggered by activation of the members of caspase protease family [14-16]. Activation of caspases during apoptosis converts the inactive, proenzyme forms of caspases into the active, processed forms which in turn cleave downstream substrates, leading to biochemical events such as DNA fragmentation [17]. Caspase-3 has been implicated in playing a critical role during apoptosis. Although many molecular pathways are involved in the apoptosis-regulatory mechanism, evidence suggests that the cell cycle and DOXinduced cell death may be involved.

In this study, we showed that the over-expression of p65 remarkably decreased the cytotoxic effect of DOX on Human Pancreatic Carcinoma (PANC1) cells, correlating with the increasing of induction for cytoplasmic $\mathrm{p} 21$. In contrast, the reduction of $\mathrm{p} 65$ by knock-down of p65 enhanced the cytotoxic effect of DOX treatment due to the increase of activation of caspase-3. We also documented that Induction of p65 enhanced the p53mediated cell death response to DOX in PANC1 cells.

\section{Methods}

\section{Cells and transfection}

Human Pancreatic Carcinoma (PANC1) cells were cultured in DMEM medium supplemented with 10\% FBS (Hyclone, logan, UT). Myc-p21-pcDNA4 and pcDNA4p53 expression vector were generated as described ref7.
ShRNA sequence against p65 (CAA AAA AAG GTC ATG GAC GGT CTA TCT CTT GAA TAG ACC GTC CAT GAC CTTT and TCC CAA AGG TCA TGG ACG GTC TAT TCA AGA GAT AGA CCG TCC ATG ACC TTTT) were clone into pSUPER-EGFP1 constructs (OligoEngine, Seattle, WA). The pSUPER-Scramble plasmid (gat ccc cTT CTC CGA ACG TGT CAC GTt tca aga gaA CGT GAC ACG TTC GGA GAA ttt ttg gaa a) was used as the nonsense control [18]. Cells were transiently transfected with expression constructs using Lipofectamine 2000 reagent (Invitrogen, Carisbad, CA).

\section{Measurement of cell death}

Cells were seeded into 96-well plates and transiently transfected $12 \mathrm{~h}$ with expression constructs for p65, p53 or shRNA-p65, followed by DOX treatment $(2 \mu \mathrm{g} / \mathrm{ml})$ for $24 \mathrm{~h}$. Then $10 \mu \mathrm{l}$ of the CCK-8 solution was added to each well of the plates and then incubated for $2 \mathrm{~h}$ in the incubator $\left(37^{\circ} \mathrm{C}\right.$ and $\left.5 \% \mathrm{CO}_{2}\right)$. Cytotoxicity assay could be used with the CCK-8 solution according to the procedure of Cell Counting Kit-8 (Dojindo Laboratories, Tokyo, Japan). The absorbency was measured at $450 \mathrm{~nm}$ using a micro-plate reader (BioTeK).

\section{Western Blotting and Immunoprecipitation}

The nuclear and cytoplasmic extract from the cells were prepared by the Nuclear Extract Kit (Active Motif, Carlsbad, CA). The protein content of the cell lysate was determined by using the Bradford calorimetric assay method (Bio-Rad, Richmond, CA). The $40 \mu \mathrm{g}$ aliquot of cytoplasmic or nuclear lysate were resolved by $12 \%$ polyacrylamide-sodium lauryl sulfate gel electrophoresis and transferred to a Hybond-C Super membrane (Amersham, Buckinghamshire, UK). The antibodies against p65 (8242, Cell Signaling), pro-caspase-3 (9665, Cell Signaling), active-casepase-3 (9664, Cell Signaling), p53 (9282, Cell Signaling), p21 (2946, Cell Signaling), CDK2 (2546, Cell Signaling), CDK4 (2906, Cell Signaling), cyclinD1 (2922, Cell Signaling), cyclinE (4129, Cell Signaling), Myc (46-0603, Invitrogen) and GAPDH (AG019, Beyotime) were used. Then the blot was incubated with a secondary antibody, IRDye 800 conjugated affinity purified anti-mouse or anti-rabbit IgG (Rockland Immunochemical, Inc., Gilbertsville, PA) and detected with Odyssey Infrared Imaging System (LI-COR Biosciences, Nebraska, USA). For immunoprecipitation, a $500 \mu \mathrm{g}$ aliquot of cell lysate was incubated for $1 \mathrm{~h}$ at $4^{\circ}$ C with the anti-p21 anti-body (2946, Cell Signaling). Immune complexes were captured by incubation with $80 \mu \mathrm{l}$ protein G-plus-agarose (Santa Cruz Biotechnology, Santa Cruz, CA) for $10 \mathrm{~h}$, then washed several times with NP-40 buffer and lysed with loading buffer to do Western Blotting analysis. 


\section{Cell growth analysis}

Cells were washed twice with PBS, trypsin zed and resuspended in PBS containing $0.1 \%$ Triton X-100 and RNase (1 mg/ml) (Sigma, St Louis, MO). The cell suspension was incubated at $37^{\circ} \mathrm{C}$ for $30 \mathrm{~min}$. Propidium iodide (Molecular Probes, Inc. Eugene, OR) was added at a final concentration of $50 \mu \mathrm{g} / \mathrm{ml}$ and the cell suspension was kept at $4^{\circ} \mathrm{C}$ for 1 hour. The cells were filtered and the cell cycle was analyzed by flow cytometry with the FACScan system (Becton Dickinson, Franklin Lakes, NJ).

\section{mRNA expression}

Total RNA of the cells were extracted by homogenization in $1 \mathrm{~mL}$ TRIzol reagent (Invitrogen, Carlsbad CA, USA), followed by chloroform re-extraction and isopropanol precipitation. The RNA was quantified with Eppendorf Biophotometer (Eppendorf, Hamburg, Germany). $0.5 \mu \mathrm{g}$ of total RNA were reverse transcribed using PrimeScriptTM RT reagent Kit (DRR037A, Takara). Real-time PCR analysis was done using primers: Bcl-2, forward: ACC TGC ACA CCT GGA TCCAG; reverse: CTT GTG GCC CAG ATA GGCAC; cIAP-1, forward: CCT GAG CAG CTT GCA AGTGC; reverse: TGA CGG ATG AAC TCC TGTCC. The average of genes was normalized to the levels of GAPDH: forward: GGT CGT ATT GGG CGC CTG GTC ACC, reverse: CAC ACC CAT GAC GAA CAT GGG GGC.

\section{Results}

Cytoplasmic p21 induced by p65 is necessary to prevent DOX-induced cell death in PANC1 cells

$\mathrm{NF} \kappa \mathrm{B} / \mathrm{p} 65$ is a transcription factor that can protect or contribute to apoptosis [19]. To examine the effect of p65 in this study, we transfected with p65 into PANC1 pancreatic cancer cells and detected remarkably increasing of p65 expression in the cells (Figure 1A). Likewise, we used p65-targeted shRNA to silence p65 expression in PANC1 cells. As seen in Figure 1A again, cells transfected with p65-targeted shRNA showed a strong reduction of endogenous p65 expression. Then, the rate of cell survival after treatment with cytotoxic drug DOX (2 $\mu \mathrm{g} / \mathrm{ml}, 24 \mathrm{~h}$ treatment) was shown in Figure $1 \mathrm{~B}$. When the PANC1 cells were forced to express p65-targeted shRNA, the percentage of DOX-induced cell death were remarkably increased as compared to control shRNA. In contrast, over-expression of p65 reduced the DOXinduced cell death as compared to vector in PANC1 cells. These data suggested that target in reduction of p65 expression in PANC1 pancreatic cancer cells was useful, leading to the potency of DOX-treatment.

It is the known fact that the expression level of p21 increased in p53-dependent or p53-independent pathway. In previous studies, we showed that $\mathrm{p} 65$ increases $\mathrm{p} 21$ expression by binding to $\mathrm{p} 21$ promoter $\kappa \mathrm{B}$ site and p21 (waf1/cip1) is involved in DOX-induced cell death [9]. To investigate the role of p21 in this study, we assessed the cytoplasmic and nuclear levels of p21 in PANC1 cells. As shown in Figure 1C, the cytoplasmic p21 protein expression level in the cell extract was significantly elevated in PANC1 cells after transfection with p65 expression vector followed by DOX treatment for $24 \mathrm{~h}$. However, the nuclear p21 was not changed, as compared to the control vector. In contrast, both cytoplasmic and nuclear levels of p21 were not changed when PANC1 cells were forced to express p65-targeted shRNA (Figure 1C).

A family of aspartate-specific cysteine proteases (caspases) plays a pivotal role in the execution of programmed cell death. To gain insight into the involvement of caspases in DOX induced cell death in this study, we investigated the effects of p65 expression status on the activation of caspase- 3 . The protein expression of pro-caspase- 3 and active caspase- 3 was assessed by Western blotting in PANC1 cells that were treated with DOX at $24 \mathrm{~h}$ following p65 over-expression or down-regulation transfection. As shown in Figure 1C again, pro-caspase-3 was significantly elevated in PANC1 cells after transfection with p65 expression vector followed by DOX treatment, as compared to control vector. More, activation of caspase- 3 protease cleavage after DOX treatment was suppressed in this condition. In contrast, when the cells were transfected with p65targeted shRNA, the activation of caspase- 3 was increased (Figure 2A). These results demonstrated that activation of caspase-3 was associated with DOXinduced cell death in PANC1 cells (Figure 1B and 1C).

\section{Pro-caspase-3 physically associated with cytoplasmic p21 induction by $\mathrm{p} 65$ in PANC1 cells}

The results described above suggest that cytoplasmic p21 induced by p65 prevented cell death induced by DOX treatment in PANC1 cells. We then examined whether there is a biochemical interaction between cytoplasmic p21 and pro-caspase-3 in PANC1 cells. The cytoplasmic p21 proteins were immunoprecipitated from those cells extract using an anti-p21 antibody. The immunoprecipitates were examined by Western blotting with an anti-pro-caspase- 3 antibody and an anti-p21 antibody. As shown in Figure 2A, the association between cytoplasmic p 21 and pro-caspase- 3 was found to remarkably increase when the cells were forced to transiently over-express p65, but not down-regulation of P65. To ask which parts of the p21 participate in interacting with pro-casepase- 3 . We used with wild-type p21 (1-585), the $\mathrm{N}$-terminal domain of p21 (1-372) and the C-terminal domain of p21 (373-585) expression vectors. These Myc-tagged recombinant p21 proteins were immunoprecipitated from those cells using an anti-Myc 


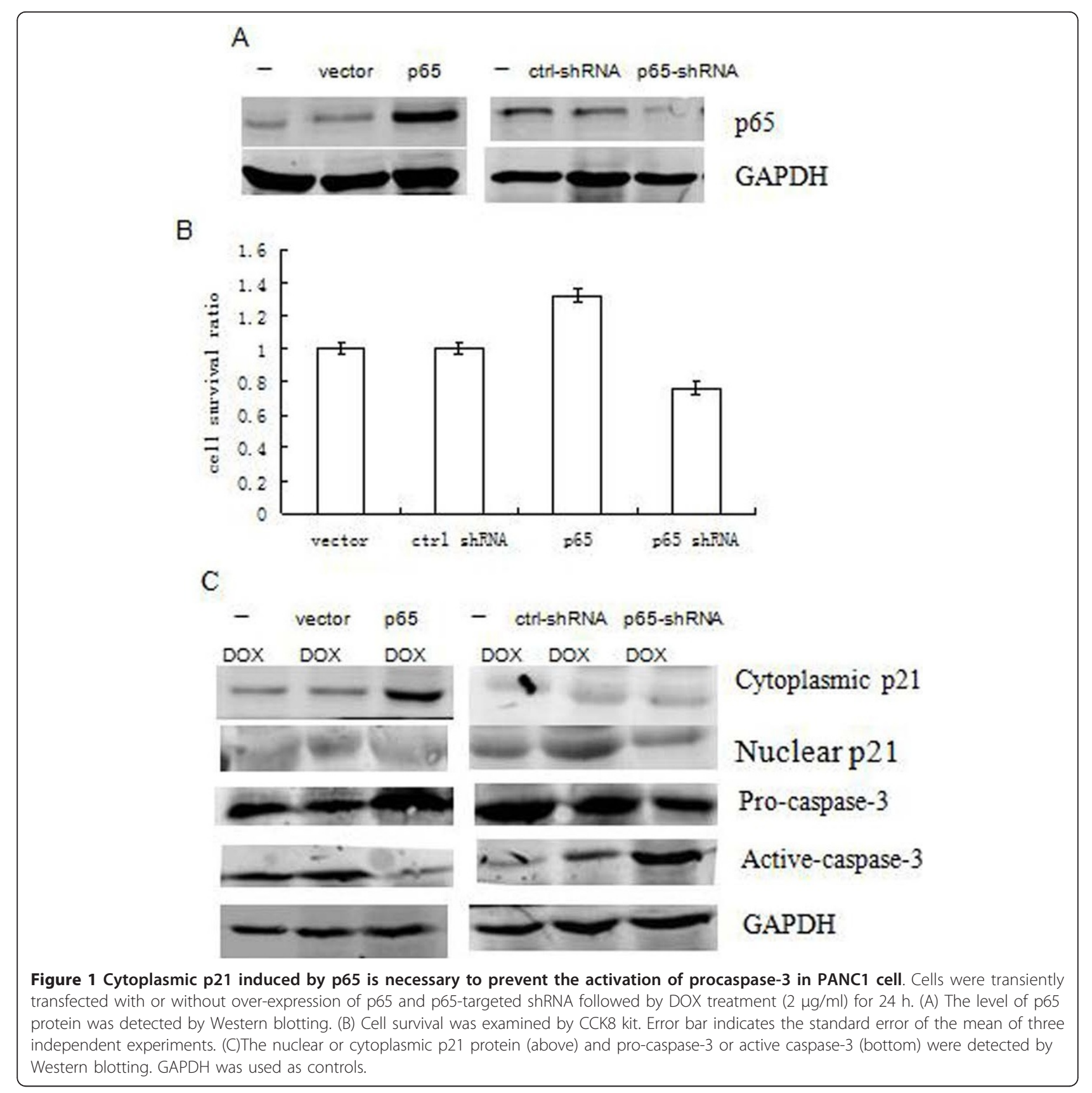

peptide antibody. The immunoprecipitates were examined by Western blotting with an anti-pro-caspase-3 antibody and an anti-p21 antibody. Wild-type p21 and the N-terminal domain were found to interact with prcaspase- 3 while the $\mathrm{C}$-terminal domain failed to associate with caspase-3 (Figure 2B). Thus, our results indicate that existing inactivated pro-caspase- 3 may prevent $\mathrm{p} 21$ translocation from cytoplasm to nucleus by physically associating with cytoplasmic p21 induced by overexpression of p65.
Next, we were interested in confirming that the effects of the down-regulation of P65 could be seen with p65regulated target genes. For these experiments, we analyzed cIAP-1 and Bcl-2, which are anti-apoptotic genes regulated by 65. The real-time PCR analysis of RNA extracted from PANC1 cells when the cells were forced to transiently over-express p65 or p65-targeted shRNA. As seen in Figure 3, cIAP-1 and Bcl-2 mRNA levels remarkably increased in PANC1 cells with the over-express p65. In contrast to these observations, down-regulation of P65 


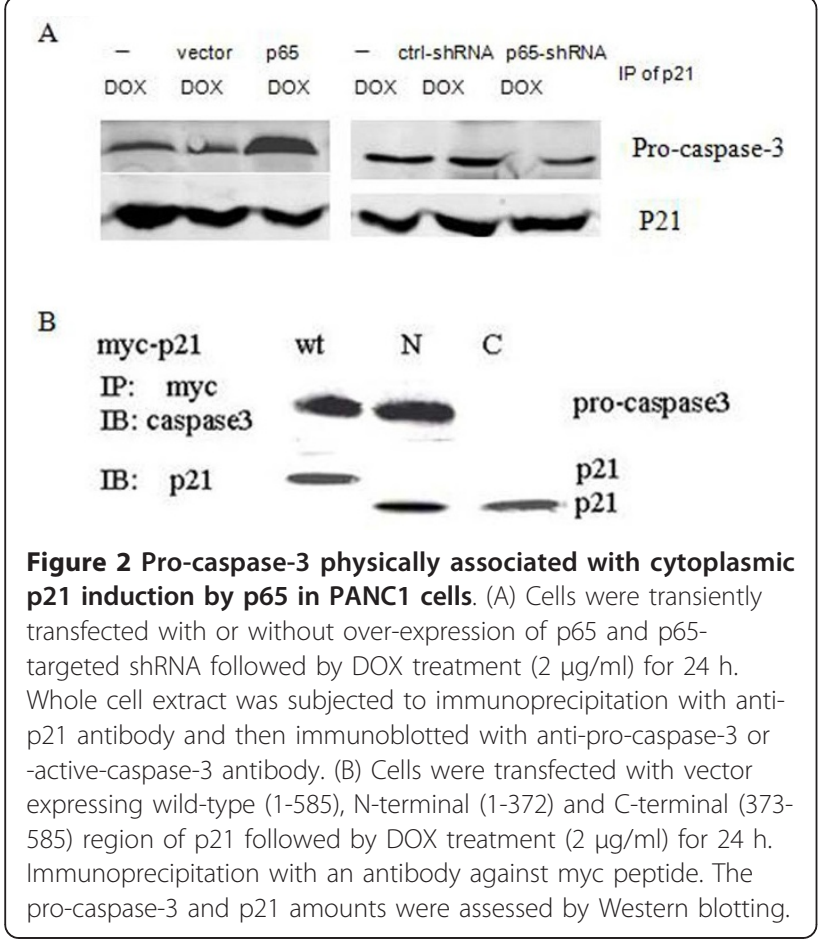

resulted in decrease in cIAP-1 and Bcl-2 mRNA levels as compared with its parental PANC1 cells (Figure 3). It led us to suggest that PANC1 cells with down-regulation of P65 were more sensitive to DOX treatment may partly due to decreasing expression of these anti-apoptotic genes.

We further sought to test some other key cell cycle regulators which also showed in Figure $1 \mathrm{C}$. Thus, we observed no remarkably change in the levels of cdk2, cdk4, cyclinE and cyclinD1 by DOX in PANC1 cells with reduction or over-expression of p65 (Figure 4A). These data was consistent with results of cell cycle analysis showed that no change in the cell cycle pattern of these cells after regulating expression of p65 (Figure 4B). Our data suggested that no clear elevation of nuclear $\mathrm{p} 21$ by $\mathrm{p} 65$ provides a survival advantage by progression cell cycle after treatment of DOX.

\section{Induction of p65 enhanced the p53-mediated cell death response to DOX in PANC1 cells}

P53 tumor suppressor is known to play an important role in mediating DNA damage agent's induced-cell death through a variety of mechanisms [20-22]. To confirm this point, we over-expressed p53 into PANC1 pancreatic cancer cells which are endogenous p53-negative (Figure 5A). Next, we used DOX to treat PANC1 (p53+ + ) cells and its parental cells for $24 \mathrm{~h}$. As shown in Figure 4B, DOX-induced cell death was remarkably higher in PANC1 $(\mathrm{p} 53++)$ cells than in its parent cells. This result suggests a requirement of p53 for DOX-induced cell death PANC1 pancreatic cancer cells.

Then we want to check p65 effects on the cells of over-expression of p53, and co-transfected with p53 and p65 into the cells. As shown in Figure 5A again, a clear elevation level of p53 or p65 was seen as compared to the control vector. Furthermore, cell survival rate was decreased after forced over-expression p53 and p65, as compared to the cells transfected with p53 alone (Figure $5 \mathrm{~B})$. These data led us to suggest that induction of p65 specifically enhanced the p53-mediated cell death response to DOX.

To investigate the role of p21 in this case, we assessed cytoplasmic and nuclear levels of p21 in these cells. As shown in Figure $5 \mathrm{C}$, the nuclear p21 protein expression level in the cell extract was significantly elevated in PANC1 cells after transfection with p53 or p53/p65 over-expression vector followed by DOX treatment for $24 \mathrm{~h}$. Moreover, the amount of cytoplasmic p21 likely corresponded to its pattern in nucleus (Figure 5C).

Next, we investigated the effects of the activation of caspase-3 on reduction cytoplasmic p21. The cytoplasmic p21 proteins were immunoprecipitated from those cells extract using an anti-p21 antibody. The immunoprecipitates were examined by Western blotting with an anti-pro-caspase- 3 antibody and an anti-p21 antibody. As shown in Figure 5C again, the association between cytoplasmic p21 and pro-caspase-3 was found to remarkably reduce when the cells were forced to transiently over-express p53 or p53/p65. These results indicate that over-expression of p53 mediated caspase- 3 activation, which may prevent pro-caspase- 3 to physically associate with cytoplasmic p 21 and then promoted it translocation from the cytoplasm to nucleus.

More, the protein expression of active caspase- 3 was assessed by Western blotting assay. As shown in Figure 5D, active-caspase- 3 was significantly elevated in PANC1 cells after transfection with $\mathrm{p} 53$ or $\mathrm{p} 53 / \mathrm{p} 65$ expression vector followed by DOX treatment, as compared to control vector.

Next, we sought to test some other key cell cycle regulators which also showed in Figure 6A. Thus, we observed remarkably reduction in the levels of cdk2, cdk4, cyclinE and cyclinD1 by DOX after transfection with p53 or p53/p65 over-expression vector. To examine the change in the cell cycle pattern of these cells, the flow cytometry was carried out (Figure 6B). As expected, in PANC1 $(\mathrm{p} 53++)$ and $(\mathrm{p} 53++/ \mathrm{p} 65++)$ cells fractions of G1-phase cells were increased from 54 and 49 to $79 \%$ and $76 \%$, indicating the cell cycle is arrested at G1-phase due to increasing of nuclear p21 expression. These data suggest data that nuclear p 21 induction by p53 promotes cell death may also due to its ability to 

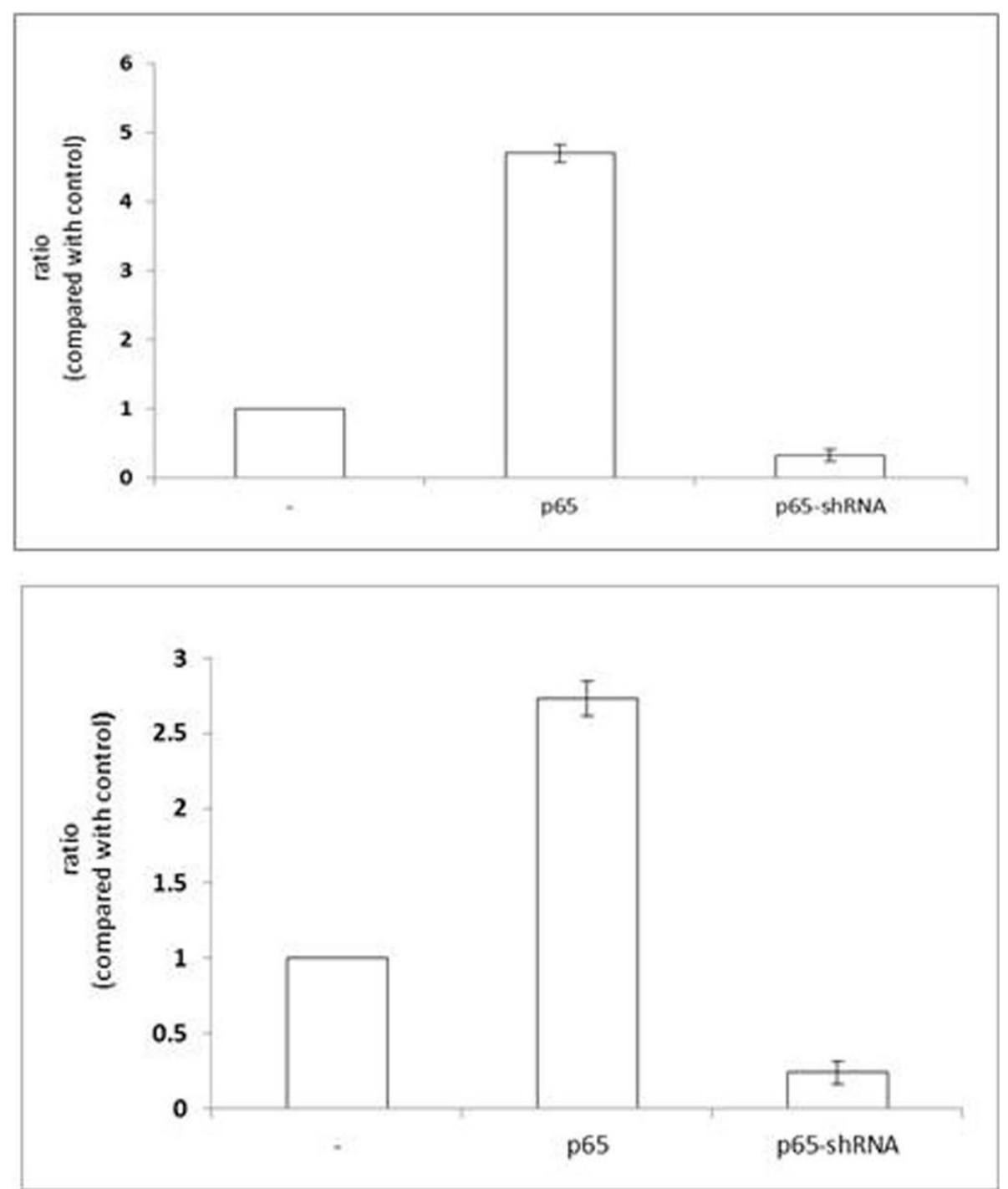

Figure 3 Down-regulation of p65 reduced expression of the anti-apoptotic genes. Cells were transiently transfected with or without overexpression of p65 and p65-targeted shRNA. Real-time PCR was performed to assess c-IAP1 (above) and BCL-2 (bottom) mRNA in the cells with GAPDH as control. Error bar indicates the standard error of the mean of three independent experiments.

stop cell cycle progression, which at least partly contributes to DOX-induced cell death in p53-dependent way.

\section{Discussion}

In this study, we showed that the over-expression of $\mathrm{p} 65$ remarkably decreased the cytotoxic effect of DOX on PANC1 cells. That correlated with the increasing of induction of cytoplasmic p21. We observed that pro-caspase-3 physically associated with cytoplasmic p21, which may prevent p21 translocation from cytoplasm. In contrast, down-regulation of p65 enhanced the cytotoxic effect to DOX treatment, due to activation of cleavage of caaspase- 3 in the cells. More, we present evidence that over-expression of $\mathrm{p} 53$ or $\mathrm{p} 53 / \mathrm{p} 65$ in the PANC1 cells were more sensitive to DOX treatment, correlated with activation of caspase- 3 and clear elevation of nuclear p21 level. Our data suggested p65 could increase p53-mediated cell death in response to DOX in PANC1 cells.

P65 is involved in the regulation of cell death in several systems [19]. In this study, we found that overexpression of p65 remarkably decreased the cytotoxic effect of DOX on PANC1 cells. In contrast, down-regulation of p65 increased DOX-induced cell death in the cells (Figure 1A and 1B). These observations suggest that a regulatory mechanism for p65 must exist to integrate and coordinate its critical cellular decision-making event. Studies have shown the existence of p21 


\section{A}

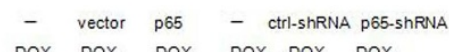
DOX DOX DOX DOX DOX DOX

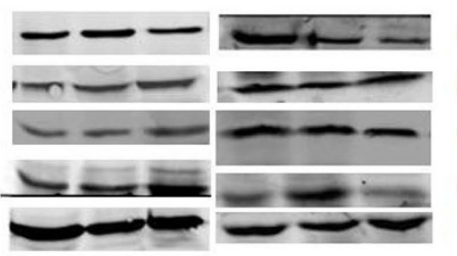

CDK2

CDK4

cyclinD1

cyclinE

GAPDH

B.

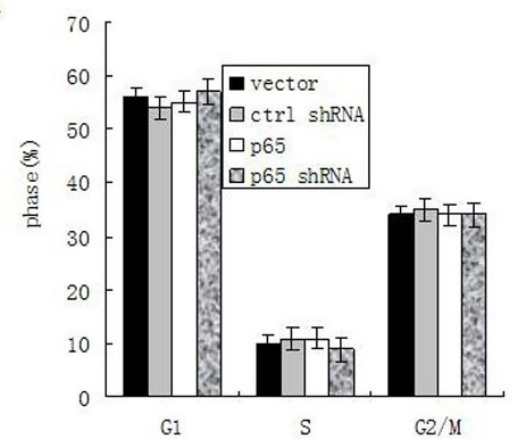

Figure 4 Change of P65 status was not related cell cycle pattern in PANC1 cells. Cells were transiently transfected with or without over-expression of p65 and p65-targeted shRNA followed by DOX treatment $(2 \mu \mathrm{g} / \mathrm{ml})$ for $\mathbf{2 4} \mathrm{h}$. (A) The level of CDK2, CDK4, and cyclinD1 or cyclinE protein were detected by Western blotting. GAPDH was used as controls. (B) Cell cycle pattern. Error bar indicates the standard error of the mean of three independent experiments.

induction in a p53-independent pathway. Our previous study indicates that DOX-induced p65 is able to bind the $\mathrm{p} 21$ promoter to activate its transactivation in the cells. Here we observed p21 induction in p65-dependent mechanism in response to DOX (Figure 1C). Thus, we suggested that induction of p21 by p65 is at least partly responsibility to decrease of DOX-induced cell death in the cells.

Genetic and biochemical studies indicate that DOXinduced cell death is triggered by activation of the members of caspase protease family $[14,16-18,22]$. Caspase-3 plays a pivotal role in the execution of DOX-induced cell death. We and other groups have reported that p65 prevents drugs-induced cytotoxic action by blocking caspases protease activation $[8,9]$. These results are consistent with our finding that down-regulation of p65 expression was preceded by $>3$-fold reduction of cIAP1 and Bcl-2 mRNA expression in PANC1 cells (Figure 3), suggesting that the decreasing expression of some anti-apoptotic genes contributed to increasing sensitive to DOX-induced cell death. Here, our results indicated that existing inactivated pro-caspase- 3 by p 65 may prevent p21 translocation from cytoplasm into nucleus by physically associating with cytoplasmic p21 (Figure $2 \mathrm{~A}$ and $2 \mathrm{~B}$ ). These data are consistent with those of recent studies showing that pro-caspase-3/p21 complex formation could resist cell death in human tumor cells [15-17]. More, our data suggested that no clear elevation of nuclear p 21 by over-expression p 65 provides a survival advantage by progression cell cycle after treatment of DOX (Figure 4A and 4B).

PANC1 pancreatic cancer cells are endogenous p53negative. Thus, our data suggest that induction of $\mathrm{p} 65$ in p53 null tumors provides a survival advantage by physically associating with procaspase- 3 and preventing p21 translocation from cytoplasm to nucleus in PANC1 cells treated with DOX. On the other hand, it is worth noting that in p53 null or defective tumors, targeting in down-regulation of p65 was found to augment an effective therapeutic response to DOX.

In this study, we showed that pancreatic tumor cells that have over-expression of p53 were more sensitive to DOX-induced cell death (Figure 5A and 5B), correlated with activation of caspase- 3 whose induction by DOX in the p53-dependent pathway (Figure 5C). The mechanism through which p53 promotes cell death is also dependent on the Apaf-1/caspase-9 pathway and involves cytochrome $\mathrm{C}$ releasing from mitochondria [20]. Several potential downstream mediators such as, CD95, Killer/DR5, PERP, p53AIP1 and Bax are involved in p53-dependent cell death $[21,22]$. Taken together, we propose that $\mathrm{p} 53$ is required for DOX-induced cell death in PANC1 pancreatic cancer cells.

Here, we observed that in response to DOX, p21 level was induced by over-expression of p53 or p53 and p65. Moreover, p53 induced caspase- 3 to cleavage to its active form after treated with DOX even occurred to co-transfection with p65. Much less physically associating with procaspase- 3 promoted p21 translocation from cytoplasm to nucleus, as evidenced by a clear elevation of nuclear p21 level induced by p53 or p53 and p 65 (Figure 5D). However, the biochemical basis of the $\mathrm{p} 21$ translocation from cytoplasm to nucleus related with caspase-3 should be clarified in the further studies.

DOX induces both cell cycle arrest and apoptosis, although the detailed mechanism whereby DOX commits cells to apoptotic program is unknown. It has been postulated that the imbalance of the cell cycle signals or failure to arrest the cell cycle may trigger the cell death program. Our previous data suggested that expression of p21 increases Gefitinib-induced cell death by inactivation of cyclin-dependent kinase (CDK) activity, which in turn blocks the cell cycle at the G1 and G2 phases [12]. The present findings here reinforced this idea by showing $\mathrm{p} 21$ 's ability of potentiality of DOX-induced cell death correlated with its inhibition of cell cycle progression after over-expression of p53 or p53/p65 (Figure 6A and $6 \mathrm{~B})$. 


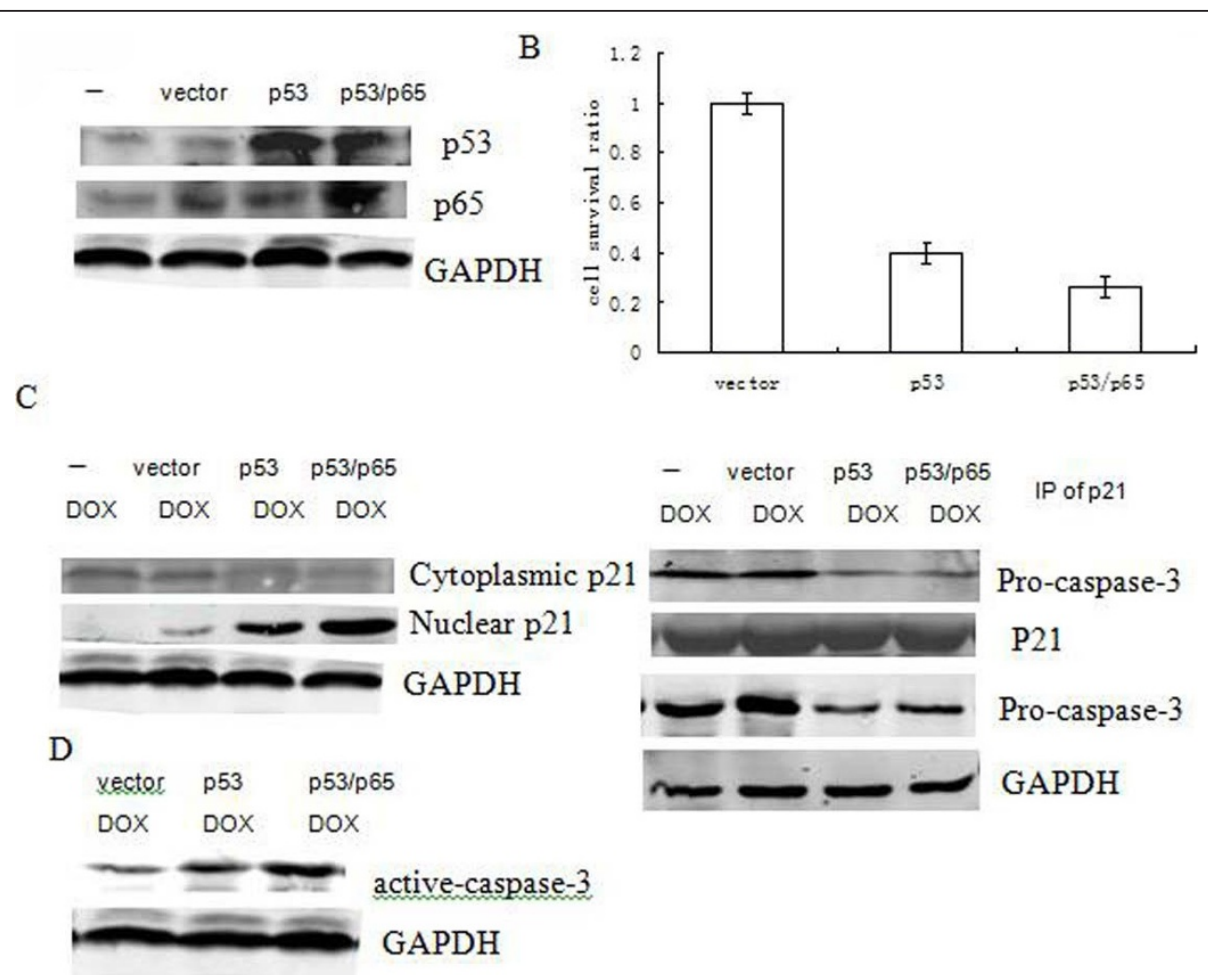

Figure 5 Induction of p65 enhanced the p53-mediated cell death response to DOX in PANC1 cells. Cells were transiently transfected with or without over-expression of p53 alone or p53 and p65 together followed by DOX treatment $(2 \mu \mathrm{g} / \mathrm{ml})$ for $24 \mathrm{~h}$. (A) The level of p53 or p65 protein was detected by Western blotting. (B) Cell survival was examined by CCK8 kit. Error bar indicates the standard error of the mean of three independent experiments. (C)The nuclear or cytoplasmic p21 protein (above) or pro-caspase-3 (bottom) were detected by Western blotting. GAPDH was used as controls. Whole cell extract was subjected to immunoprecipitation with anti-p21 antibody and then immunoblotted with anti-pro-caspase-3 antibody (middle). (D) Active-caspase-3 and active-caspase-8 protein were detected by Western blotting. GAPDH was used as controls.

P53 plays an important role in the tumor therapy. However, it is reported that more than $60 \%$ human tumors have lost their wild-type p53 function. Increasing evidence suggests that deficiency of p53 in tumors constitutes resistance to chemotherapy and anti-angiogenic treatment. Hence, efforts have been made to improve the efficacy of anti-tumor therapy on the tumors deficient in p53 expression. Here we suggested that in tumor cells that are p53-null or defeated, downregulation of p65 may well be useful, leading to the potentiality of chemotherapeutic drugs.

However, in tumors that retain wild-type p53, such targeting may be counterproductive, because p65 potentiality of p53-mediated cell death in this study. The tumor suppressor p53 inhibits cell growth through activation of cell-cycle arrest and apoptosis. Our previous data suggested that p65-induced anti-apoptotic gene expressions are much higher in p53-/- cells than in p53 $+/+$ cells. We and other groups found that the transcriptional activities of p53 and p65 are governed by their relative levels of expression: p65 inhibits p53-dependent transactivation, while p53 expression can also suppress p65 transcriptional activity $[8,9]$. P53 could limit p65mediated transactivation and this mutual repression mechanism is due to their limiting common co-activators (p300 and CBP) of transcription [23-26]. Thus, we could speculate that p 65 loss the ability to promote to induce anti-apoptotic gene expressions, then turn into pro-apoptotic process in $53+/+$ cells, which contributes to p65 augmenting an effective therapeutic response to DOX in p53-mediated way.

\section{Conclusion}

In the present study, we observed that the p21 expression level was a key player in the cell cycle and DOXinduced cell death process. The clear elevation of nuclear p 21 expression by p53 or p53 and p65 may provide a good rationale for promoting to DOX treatment in Human Pancreatic Carcinoma. Thus, we propose that it may be important in the design of therapeutic protocols that involve targeting of p21 to mediate pancreatic tumor's sensitivity to the drugs. 
A

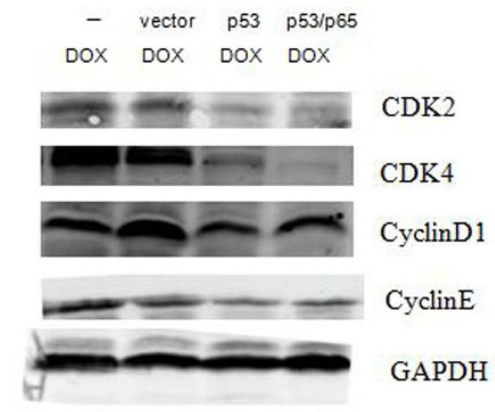

B.

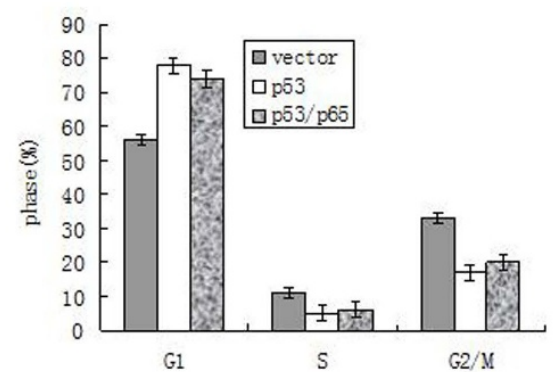

Figure 6 P53 promoted DOX-induced cell death was partly due to its ability to stop cell cycle progression. Cells were transiently transfected with or without over-expression of p53 alone or p53 and p65 together followed by DOX treatment $(2 \mu \mathrm{g} / \mathrm{ml})$ for $24 \mathrm{~h}$. (A) The level of CDK2, CDK4, cyclinD1 or cyclinE protein were detected by Western blotting. GAPDH was used as controls. (B) Cell cycle pattern. Error bar indicates the standard error of the mean of three independent experiments.

\section{Acknowledgements}

This work was supported by the Natural Science Foundation of China (81071740), Shanghai Science Foundation (10ZR1406300) and Yangfan Project of Tongji University School of Medicine (2010YF04).

\section{Author details}

${ }^{1}$ The Third General Surgery Department, Zhanghai Hospital, Second Military Medical University, 168 Zhanghai Road, Shanghai 200433, China.

${ }^{2}$ Department of Pathology, Zhongshan Hospital, Fudan University, Shanghai, China. ${ }^{3}$ Laboratory of Cancer Research, Tongji University School of Medicine, 1239 Siping Road, Shanghai 200092, China.

\section{Authors' contributions}

YQZ carried out the cell culture and transfection experiments and drafted the manuscript. GL carried out cell growth and cell death experiments, and contributed to the experiments plans. YJ participated in constructing all those expression plasmids. CL carried out the Western blotting and Immunoprecipitation assay. JPZ carried out Real-time PCR experiment and revised part of manuscript. YJL designed of the study and wrote the manuscript. All authors read and approved the final manuscript.

\section{Competing interests disclosure}

The authors declare that they have no competing interests.

Received: 2 November 2011 Accepted: 4 February 2012 Published: 4 February 2012

\section{References}

1. Hezel AF, Kimmelman AC, Stanger BZ, Bardeesy N, Depinho RA: Genetics and biology of pancreatic ductal adenocarcinoma. Genes Dev 2006, 20:1218-49.

2. Jemal A, Siegel R, Ward E, Hao Y, Xu J, Murray T, Thun MJ: Cancer statistics. CA Cancer J Clin 2008, 58:71-96.

3. Whiteside ST, Ernst MK, Lebail O, Laurent-Winter C, Rice N, Israe AA: N- and C-terminal sequences control degradation of MAD3/I kappa $B$ alpha in response to inducers of NF-kappa B activity. Mol Cell Biol 1995, 15:5339-5345.

4. El-Deiry WS, Tokino T, Velculescu VE: WAF1, a potential mediator of p53 tumor suppression. Cell 1993, 75:817-825.

5. Waldman T, lengauer $C$, Kinzler KW: Uncoupling of s-phase and mitosis induced anticancer agents in cell lacking P21WAF1/CIP1. Nature 1996, 381:713-716.

6. Lu YJ, Yamagishi N, Yagi T, Takebe H: Mutated p21 (WAF1/CIP1/SDT1) lacking CDK-inhibitory activity fails to prevent apoptosis in human colorectal carcinoma cell. Oncogene 1998, 16:705-712.

7. Lu YJ, Tatsuka T, Takebe H, Yagi T: Involvement of cyclin-dependent kinases in doxorubicin-induced apoptosis in human tumor cells. $\mathrm{Mol}$ Carcinog 2000, 29:1-7.

8. Ma SL, Tang JJ, Feng JG, Xu Y, Yu X, Deng Q, Lu Y: Induction of p21 (waf1) by p65 in p53 null cells treated with Doxorubicin. Biochimica et Biophysica Acta 2008, 1783:935-940.

9. Webster GA, Perkins ND: Transcriptional Cross Talk between NF- kappa B and p53. Mol Cell Biol 1999, 19:3485-3495.

10. Hellin AC, Bentires-Alj M, Verlaet M, Benoit V, Gielen J, Bours V, Merville MP: Roles of Nuclear Factor-kB, p53, and p21/WAF1 in Daunomycin-Induced Cell Cycle Arrest and Apoptosis. J Pharmacol Exp Ther 2000, 295:870-878.

11. Ji Y, Ma SL, Zhang YP, Tang JJ, Wu YM, Lu YJ: A combined treatment TNFa/Gefitinib alleviates the resistance to Gefitinib in PC-9 cells with acquired resistance to Gefitinib. Anti-Cancer Drug 2009, 20:832-827.

12. Zhao YF, Wang CR, Wu YM, Ma SL, Ji Y, Lu YJ: P21 (waf1/cip1) is required for non-small cell lung cancer sensitive to Gefitinib Treatment. Biomedicine and pharmacotherapy 2011, 5:151-156.

13. Wu H, Chen Y, Wang ZY, Li W, Li JQ, Zhang L, Lu YJ: Involvement of p21 (waf1) in merlin deficient sporadic vestibular schwannomas. Neuroscience 2010, 170:149-155.

14. Steller H: Mechanisms and Genes of Cellular Suicide. Science 1995, 267:1445-1449.

15. Suzuki A, Kawano H, Hayashida M, Hayasaki Y, Tsutomi Y, Akahane K: Procaspase 3/p21 complex formation to resist Fas-mediated cell death is initiated as a result of the phosphorylation of $\mathrm{p} 21$ by protein kinase $A$. Cell death Differ 2000, 7:721-728.

16. Suzuki A, Tsutomi Y, Miura M, Akahane K: Caspase 3 inactivation to suppress Fas-mediated apoptosis identification of binding domain with p21 and inactivation machinery by p21. Oncogene 1998, 18:1239-1244.

17. Chen J, Peters R, Saha P, Lee P, Theodoras A, Pagano M, Wagner G, Dutta A: Procaspase 3/p21 complex formation to resist Fas-mediated cell death is initiated as a result of the phosphorylation of $\mathrm{p} 21$ by protein kinase A. Nucleic Acids Res 1996, 24:1727-1733.

18. Pager $C T$, Dutch RE: Cathepsin $L$ is involved in proteolytic processing of the Hendra virus fusion protein. J Virol 2005, 79:12714-12720.

19. Lin B, Williams-skipp C, Tao YX, Schleicher MS, Cano LL, Duke RC, Scheinman Rl: NF-kappaB functions as both a proapoptotic and antiapoptotic regulatory factor within a single cell type. Cell Death Differ 1999, 6:570-582.

20. Wu GS, Ding ZH: Caspase 9 is required for p53-dependent apoptosis and chemo-sensitivity in a human ovarian cancer cell line. Oncogene 2000, 21:1-8.

21. Nakano K, Vousden KH: A novel proapoptotic gene is induced by $\mathrm{p} 53$. Mol Cell 2001, 7:683-694.

22. Zhang L, Yu J, Park BH, Kinzler KW, Vogelstein B: Role of BAX in the apoptotic response to anticancer agents. Science 2002, 290:989-992.

23. Avantaggiati ML, Ogryzko V, Gardner K, Giordano A, Levine AS, Kelly K Recruitment of p300/CBP in p53-dependent signal pathways. Cell 1997, 89:1175-1184.

24. Kawauchi J, Zhang C, Nobori K, Hashimoto Y, Adachi MT, Noda A, Sunamori M, Kitajima S: Transcriptional repressor activating transcription factor 3 protects human umbilical vein endothelial cells from tumor 
necrosis-a-induced apoptosis though down-regulation of $\mathrm{p} 53$ transcription. J Biol Chem 2002, 277:39025-39034.

25. Huang WC, Ju TK, Hung MC, Chen CC: Phosphorylation of CBP by IKKapromotes cell growth by switching the binding preference of CBP from p53 to NF-KB. Mol Cell 2003, 26:75-87, 2003.

26. Ryan KM, Ernst MK, Rice NR, Vousden KH: Role of NF-kappa-B in p53mediated programmed cell death. Nature 2000, 404:892-897.

doi:10.1186/1423-0127-19-15

Cite this article as: Zhou et al:: Cytoplasmic p21 induced by p65

prevents doxorubicin-induced cell death in pancreatic carcinoma cell line. Journal of Biomedical Science 2012 19:15.

Submit your next manuscript to BioMed Central and take full advantage of:

- Convenient online submission

- Thorough peer review

- No space constraints or color figure charges

- Immediate publication on acceptance

- Inclusion in PubMed, CAS, Scopus and Google Scholar

- Research which is freely available for redistribution

Submit your manuscript at www.biomedcentral.com/submit
C Biomed Central 